\title{
Sensibilidade e especificidade da ressonância magnética multiparamétrica na detecção do câncer de próstata
}

\author{
Sensitivity and specificity of multiparametric magnetic resonance in the detection of \\ prostate cancer
}
Sensibilidad y especificidad de la resonancia magnética multiparamétrica en la detección del cáncer de próstata

Heitor Maia Henriques Malveira ${ }^{1 *}$, Késia Lorrany Porto de Souza ${ }^{1}$, Luiz Filipe Lima Laranjeira Pagani $^{1}$, Rayme Maia Martins ${ }^{1}$, Marcos Vinícius Macedo de Oliveira ${ }^{1}$, Maria Fernanda Borges Abreu ${ }^{1}$.

\section{RESUMO}

Objetivo: Analisar a sensibilidade e especificidade da ressonância magnética na detecção de câncer de próstata. Métodos: Neste trabalho, foi realizado um estudo transversal, quantitativo, de exames de ressonância magnética multiparamétrica de próstata (RM-rp), nos meses de janeiro a fevereiro de 2020, no banco de dados em um grupo de clínicas de grande porte da cidade de Montes Claros - MG e região. Foram analisados 2200 prontuários de pacientes assistidos na clínica, com idades entre 45 e 75 anos. A coleta envolveu casos que apresentaram na RM-rp da próstata lesões suspeitas de câncer de próstata através do critério PI-RADS (com escores entre 3-5) e PSA total pré-teste presente. Resultados: Pode-se inferir que o uso da RM associada ao PSA $\geq 4 \mathrm{ng} / \mathrm{dl}$ aumenta a fidedignidade do diagnóstico por aumentar a sensibilidade e diminuir casos de falso positivo. Além disso, essa associação maximiza o benefício desse método como exame de triagem, pois também diminui possíveis custos desnecessários e indesejadas iatrogenias. Conclusão: A RM-rp eleva os dados de sensibilidade e especificidade quando comparado ao PSA de maneira isolada, mas não deve ser o primeiro exame a ser solicitado e sim caso haja uma alteração de PSA.

Palavras-chave: Câncer de próstata, Ressonância magnética, Biópsia.

\begin{abstract}
Objective: To analyze the sensitivity and specificity of magnetic resonance in the detection of prostate cancer. Methods: In this work, a cross-sectional, quantitative study of prostate multiparametric magnetic resonance imaging (RM-rp) was performed, from January to February 2020, in the database of a group of large clinics in the city de Montes Claros - MG and region. 2200 medical records of patients seen at the clinic, aged between 45 and 75 years, were analyzed. The collection involved cases that presented lesions suspected of prostate cancer in the prostate MR-rp using the PI-RADS criterion (with scores between 3-5) and total pre-test PSA present. Results: It can be inferred that the use of MRI associated with PSA $\geq 4 \mathrm{ng}$ / dl increases the reliability of the diagnosis by increasing sensitivity and decreasing cases of false positives. In addition, this association maximizes the benefit of this method as a screening test, as it also decreases possible unnecessary and unwanted iatrogenic costs. Conclusion: RM-rp raises the sensitivity and specificity data when compared to PSA in isolation, but it should not be the first test to be requested, but if there is a change in PSA.
\end{abstract}

Keywords: Prostate cancer, Magnetic resonance imaging, Biopsy.

${ }^{1}$ Centro Universitário FipMoc (UNIFIPMOC), Montes Claros - MG. *E-mail: heitor.mhm@gmail.com 


\section{RESUMEN}

Objetivo: Analizar la sensibilidad y especificidad de la resonancia magnética en la detección del cáncer de próstata. Métodos: En este trabajo, se realizó un estudio transversal cuantitativo de la resonancia magnética multiparamétrica de próstata (RM-rp), de enero a febrero de 2020, en la base de datos de un grupo de grandes clínicas de la ciudad de Montes Claros - MG y región. Se analizaron 2200 registros médicos de pacientes atendidos en la clínica, con edades comprendidas entre 45 y 75 años. La colección incluyó casos que mostraron sospecha de lesiones de cáncer de próstata en el MR-rp de próstata utilizando el criterio PI-RADS (con puntajes entre 3-5) y el PSA total previo a la prueba. Resultados: Se puede inferir que el uso de MRI asociado con PSA $\geq 4 \mathrm{ng}$ / dl aumenta la confiabilidad del diagnóstico al aumentar la sensibilidad y disminuir los casos de falsos positivos. Además, esta asociación maximiza el beneficio de este método como prueba de detección, ya que también disminuye los posibles costos iatrogénicos innecesarios y no deseados. Conclusión: RM-rp aumenta los datos de sensibilidad y especificidad en comparación con el PSA de forma aislada, pero no debería ser la primera prueba que se solicita, pero si hay un cambio en el PSA.

Palabras clave: Cáncer de próstata, Resonancia magnética, Biopsia.

\section{INTRODUÇÃO}

A próstata é uma glândula pélvica cuja função se resume a armazenar e secretar um líquido alcalino constituinte do sêmen (MOORE KI, et al., 2018). O câncer de próstata (CP), decorrente da disfunção da regulação da multiplicação celular gerando populações de células anormais e de crescimento incontrolável, é a neoplasia mais comum no sexo masculino. E, apesar de bom prognóstico, com taxa de sobrevida de $80 \%$ em cinco anos, esse câncer representa $13,4 \%$ do número total de mortes masculinas no Brasil (SANTOS M, 2018).

Entre os fatores de risco identificados para o câncer de próstata está o avanço da idade, visto que tanto a incidência quanto a mortalidade aumentam após os 50 anos, envolvendo aproximadamente $80 \%$ dos homens aos 80 anos (JEMAL A, et al., 2015 e SANTOS M, 2018). Além disso, outra associação positiva que agrega risco de desenvolvimento dessa neoplasia é o histórico familiar positivo em primeiro grau; esse aspecto acrescenta aproximadamente $25 \%$ de chance de o indivíduo desenvolver a doença (INSTITUTO NACIONAL DE CÂNCER, 2019).

O balanço entre benefícios e riscos é o principal ponto analisado para realização de rastreamento de câncer, sendo uma estratégia dirigida a um grupo populacional específico em que a prática seja mais favorável, impactando positivamente na redução da mortalidade (INSTITUTO NACIONAL DE CÂNCER, 2019). O rastreio deve ser iniciado a partir de 50 anos com avaliação individualizada e naqueles pacientes considerados de grupo de risco, como raça negra ou com parentes de primeiro grau com câncer de próstata devem começar aos 45 anos (SOCIEDADE BRASILEIRA DE UROLOGIA, 2019).

A suspeita do câncer de próstata é feita pelo toque digital da próstata e por meio de medidas do antígeno próstata específico sérico (MOTTET N, et al., 2017). Tumores de próstata identificados pelo toque retal, em geral, já possuem um tamanho relativamente grande, o que implica maior chance de doença avançada, assim, em caso de positividade, é mandatório a realização de biópsia (MOTTET N, et al., 2017).

O Antígeno Prostático Específico (PSA) é um marcador sérico de dano prostático, podendo circular em duas formas bioquímicas, livre ou associada com a1-antiquimiotripsina. A dosagem rotineira de PSA é muito controversa uma vez que sua elevação não é exclusiva para neoplasia prostática, uma vez que esse antígeno não é produzido especificamente pela célula cancerosa e sim pelas células epiteliais da próstata, podendo estar alterado por outras patologias, como a hiperplasia benigna da próstata e a prostatite, assim como após a ejaculação e uma realização de cistoscopia (ASSOCIAÇÃO EUROPEIA DE UROLOGIA, 2018).

Entretanto, quanto maior a dosagem de PSA, maior a probabilidade de CP. Quando o PSA estiver acima de $10 \mathrm{ng} / \mathrm{ml}$, há indicação formal para biópsia, porém ainda não há consenso a respeito do ponto de corte considerado ideal, pois assim como já demonstrado, 15,2\% dos homens que tiveram resultados inferiores a 
$4 \mathrm{ng} / \mathrm{dl}$, após um toque retal sem suspeita clínica de tumor, chegaram a evoluir para doença de alto grau, fazendo com que o valor de corte para indicar a biópsia fosse revisto e idealmente, levando em consideração a velocidade do PSA e a relação PSA livre/total (ASSOCIAÇÃO EUROPEIA DE UROLOGIA, 2018).

A biópsia de próstata por via transretal é considerada um dos procedimentos urológicos mais empregados no Ocidente. Apesar da utilidade diagnóstica, essa técnica não é desprovida de limitações que incluem elevada taxa de resultados falsos negativos, estratificação de risco imprecisa e detecção de pequenos tumores clinicamente insignificantes (PARKER C, et al., 2015).

Por outro lado, a ressonância magnética de próstata demonstra-se com crescente relevância para prática urológica, possibilitando maior visualização da próstata e de suas alterações, se comparada com a ecografia prostática transretal (BJURLIN M, et al., 2014).

Atualmente, desenvolveram-se sequências funcionais de Ressonância Magnética (RM) que permitem a obtenção de informações acerca da fisiologia dos tecidos, dentre elas, a mais utilizada para estudo da próstata, a ressonância magnética multiparamétrica (RM-mp), associa sequências morfológicas ponderadas em T1 e T2 com, no mínimo, duas sequências funcionais, como a técnica de difusão, realce dinâmico após injeção de contraste e a espectroscopia (KASIVISVANATHAN V, et al., 2018).

Tal associação é responsável pelo aumento da sensibilidade e especificidade para detecção de CP para, respectivamente, $74 \%$ e $88 \%$, além de aumentar a probabilidade de diagnóstico de tumores clinicamente insignificantes e reduzir a necessidade de biópsias adicionais (KASIVISVANATHAN V, et al., 2018). Além disso, a RM-mp apresenta um valor preditivo negativo elevado para o câncer prostático, sendo favorável sua utilização como ferramenta de triagem e avaliação da doença, mesmo tendo a especificidade e o valor preditivo positivo considerados baixos (MORAES M, 2017).

Com o objetivo de reduzir a variação de interpretações dos resultados obtidos pela RM, foi criado uma classificação para padronização dos achados, denominada PI-RADS. Essa classificação varia de 1 a 5, sendo que PI-RADS 1 significa que há uma lesão significante altamente improvável, 2 uma lesão significante improvável, 3 significante e equívoca com necessidade de biópsia dirigida a partir desse escore, 4 significante provável e 5 significante altamente provável (COLÉGIO AMERICANO DE RADIOLOGIA, 2015).

Uma vez diagnosticada a neoplasia de próstata, surge a necessidade de realização de exames laboratoriais e de imagem com intuito de obter o estadiamento da doença para estabelecer a linha de tratamento mais efetiva e o prognóstico do paciente. Os parâmetros utilizados nessa avaliação são os valores de PSA, o escore de Gleason e o estádio de acordo com a American Joint Committee on Cancer, que juntos conseguem classificar em pacientes de baixo, intermediário e alto risco (FAY et al., 2017).

O escore de Gleason tem como objetivo informar sobre a provável taxa de crescimento do tumor bem como sua probabilidade de disseminação, ajudando também na determinação do tratamento mais eficaz para o paciente. As células alteradas são comparadas às células prostáticas normais e quanto maiores forem as diferenças entre elas, deduz-se que o tumor será mais agressivo e mais rápido em disseminação. Essa escala varia de 1 a 5 , sendo a forma menos agressiva o grau 1 .

O escore total dessa classificação é dado pelo patologista, que gradua de 1 a 5 as duas áreas mais frequentes em apresentação de células doentes, somado posteriormente os resultados, que variam, portanto de 2 a 10. Os melhores prognósticos acompanham os menores valores (FAY et al., 2017). Dessa forma, esse trabalho tem como objetivo analisar a sensibilidade e especificidade da ressonância magnética na detecção de carcinoma de próstata.

\section{MÉTODOS}

\section{Caracterização da pesquisa}

Neste trabalho, foi realizado um estudo transversal, quantitativo, de exames de ressonância magnética multiparamétrica de próstata (RM-rp) em um grupo de clínicas de grande porte da cidade de Montes Claros MG e região. 


\section{População}

Foram investigados cerca de 2200 prontuários de pacientes assistidos na clínica. Foram selecionados para a pesquisa aqueles prontuários que atenderam aos seguintes critérios de inclusão: idade entre 45-75 anos, presença de PSA pré-exame, ressonância multiparamétrica da próstata pelo equipamento Achieva 3.0 Tesla da marca Philips, com antena de coluna de 16 canais sem body phased array, e laudo histopatológico. Foram excluídos da pesquisa prontuários com dados inconsistentes ou incompletos.

\section{Procedimentos e instrumentos}

Foram avaliados, nos meses de janeiro a fevereiro de 2020, no banco de dados da clínica, resultados das ressonâncias magnéticas multiparamétrica de 2200 pacientes. A coleta foi realizada com orientação do administrador quanto ao uso do sistema e acompanhada por um membro do setor técnico de informatização da empresa. Foram acessados no sistema da clínica dados como: nome do paciente, idade, laudo da ressonância multiparamétrica de próstata, resultado do PSA sérico (total e refinado) e dado do histopatológico em classificação Gleason.

Houve a seleção dos casos que apresentarem na RM-rp da próstata lesões suspeitas de CP através do critério PI-RADS (com escores entre 3-5) e PSA total pré-teste presente. Ao final, todos os pacientes que tiverem áreas suspeitas e biópsias guiadas pelo achado na RM terão seu histopatológico com grau Gleason analisado, sendo este avaliado de forma qualitativa para o câncer (presença ou não).

\section{Análise dos dados}

Todas as informações foram tabuladas no software Microsoft Exce ${ }^{\circledR}$ e validadas em uma calculadora da Biblioteca Virtual da Saúde (BVS). Os níveis de PSA sérico (total) foram comparados aos resultados dos exames histopatológicos (em Gleason) e da RM-rp (em PI-RADS), a fim de se tentar correlacionar possíveis níveis de PSA ao grau de sugestão de malignidade encontrado.

A partir das características descritas no histopatológico e em conjunto com a classificação PI-RADS, também foi possível compará-las e determinar o grau de equivalência entre os resultados dos exames. $O$ resultado do histopatológico (qualitativo) foi considerado o Golden-standard, e os da RM-rp então foram comparados a eles a fim de ser atribuído para cada exame de ressonância o resultado do laudo de um verdadeiro positivo (VP), verdadeiro negativo (VN), falso positivo (FP) ou falso negativo (FN) para lesões malignas e/ou benignas. Com os resultados, foi possível analisar o desempenho da RM-rp considerando o resultado do histopatológico. Essas variáveis foram calculadas a partir das equações dispostas na Tabela 1.

Tabela 1 - Critérios utilizados na análise dos resultados.

\begin{tabular}{ll}
\hline Critério & Fórmula \\
\hline Sensibilidade & {$[\mathrm{VP} /(\mathrm{VP}+\mathrm{FN})]$} \\
Especificidade & {$[\mathrm{VN} /(\mathrm{VN}+\mathrm{FP})]$} \\
Acurácia & {$[(\mathrm{VP}+\mathrm{VN}) /(\mathrm{VP}+\mathrm{VN}+\mathrm{FP}+\mathrm{FN})]$} \\
Valor Preditivo Positivo (VPP) & $(\mathrm{VP} / \mathrm{VP}+\mathrm{FP})$ \\
Valor Preditivo Negativo (VPN) & $(\mathrm{VN} / \mathrm{VN}+\mathrm{FN})$ \\
\hline
\end{tabular}

Fonte: Malveira HMH, et al., 2020.

\section{Aspectos Éticos}

A pesquisa foi aprovada pelo Comitê de Ética em Pesquisa para sua execução (parecer 3.763.804/2020), seguiu as normas do Conselho Nacional de Saúde (resolução 466/12).

\section{RESULTADOS E DISCUSSÃO}

Após coleta e análise de dados dos cerca de 2200 prontuários, foram considerados aptos ao estudo 200 pacientes, que se enquadraram nos critérios pré-estabelecidos na metodologia deste trabalho. Na literatura, encontramos o nível de corte para investigação prostática em $4,0 \mathrm{ng} / \mathrm{mL}$, com uma zona de dúvida entre 4,0 $\mathrm{ng} / \mathrm{mL}$ e $10,0 \mathrm{ng} / \mathrm{mL}$, como apresentado nos trabalhos de Mulhem E, et al. (2015), Stephen RE, et al. (2018) e Gonçalves TN e Mella Junior SE (2007). O trabalho de Castro HAS, et al. (2011) defende valores menores, partindo de 2,6ng/mL, a fim de aumentar a sensibilidade do método. 
No presente estudo, analisamos 200 coletas de PSA em pacientes em investigação de câncer de próstata, conforme a Tabela 2. Desses 200, 158 tiveram PSA de valores iguais ou superiores a 4,0 ng/mL. Entretanto, apenas 82 desses tiveram suas biópsias positivadas, e 13 pessoas das $42 \mathrm{com}$ dosagem de PSA inferior a $4,0 \mathrm{ng} / \mathrm{mL}$ tiveram biópsias positivadas. Isso representa uma sensibilidade de $86,32 \%$ e especificidade de $27,62 \%$. Tais valores são semelhantes àqueles encontrados na literatura em Mulhem $E$ et al. (2015) e Furini AAC, et al. (2017). Os valores preditivos negativos e positivos para o PSA igual ou superior a $4,0 \mathrm{ng} / \mathrm{ml}$ foram, respectivamente, de $69,05 \%$ e $51,90 \%$.

Quando analisamos o critério de corte em 10,0 ng/mL (fugindo, dessa maneira, da zona considerada de dúvida), encontramos 38 pacientes com critério positivo. Destes, 23 tiveram a confirmação da neoplasia na análise histológica. Outros 72 pacientes que tiveram PSA abaixo de 10,0 ng/mL tiveram confirmação da biópsia, apesar do PSA estar num nível considerado "de dúvida". Isso representa um aumento expressivo na especificidade do corte, em $85,71 \%$, porém representa uma queda igualmente importante na sensibilidade, em $24,21 \%$. Os valores preditivos negativos e positivos para o PSA $>10,0 \mathrm{ng} / \mathrm{ml}$ foram, respectivamente, de $55,56 \%$ e $60,53 \%$.

Tabela 2 - Distribuição dos casos prostáticos, consoante o nível de PSA e o diagnóstico histopatológico de benignidade ou malignidade.

\begin{tabular}{cccc}
\hline & \multicolumn{3}{c}{ Diagnóstico Histopatológico } \\
\hline \multirow{2}{*}{ PSA } & Benigno & Maligno & Total \\
\cline { 2 - 4 } & $\mathbf{N}(\%)$ & $\mathbf{N}(\%)$ & $\mathbf{N}(\%)$ \\
\hline$<4 \mathrm{ng} / \mathrm{ml}$ & $29(69,0)$ & $13(31,0)$ & $42(21,0)$ \\
$\geq 4 \mathrm{ng} / \mathrm{ml}$ & $76(48,1)$ & $82(51,9)$ & $158(79,0)$ \\
$\geq 10 \mathrm{ng} / \mathrm{ml}$ & $15(39,5)$ & $23(60,5)$ & $38(19,0)^{*}$ \\
Total & $105(52,5)$ & $95(47,5)$ & $200(100)$ \\
\hline
\end{tabular}

Legenda: *Há membros comuns entre os pacientes com PSA $\geq 4 \mathrm{ng} / \mathrm{ml}$ e $\geq 10 \mathrm{ng} / \mathrm{ml}$. Fonte: Malveira $\mathrm{HMH}$, et al., 2020.

Considerando que o PSA é um exame de rastreio do câncer que pode indicar biópsias, apesar de os níveis de PSA > 10ng $/ \mathrm{mL}$ significarem uma maior especificidade e um maior risco da suspeita clínica se confirmar, o número de falsos negativos nesse corte preocupa e limita sua utilização ao induzir um número considerável de subdiagnósticos. Quando analisamos o corte a partir de $4,0 \mathrm{ng} / \mathrm{mL}$ diminuímos essa limitação, porém aumentamos o número de pacientes expostos a procedimentos invasivos de maneira desnecessária.

Isso indica que, isoladamente, o PSA total $4,0 \mathrm{ng} / \mathrm{mL}$ não é uma boa ferramenta para indicar biópsias de próstata, o que muda quando consideramos os níveis a partir de $10 \mathrm{ng} / \mathrm{mL}$, que tem uma especificidade maior. Entretanto, não devemos considerar a biópsia somente a partir destes níveis, pois valores inferiores também podem representar a presença do câncer (JEMAL A, et al., 2015).

Além do mais, nosso estudo evidenciou uma parcela considerável (13/200) de casos com PSA abaixo do corte e biópsias positivas, o que também é relatado na literatura em um estudo de caso de Alves LS e Assis BPS (2007). Isso poderia ser minimizado ao utilizar o corte de $2,6 \mathrm{ng} / \mathrm{mL}$ para investigação, como visto em Castro HAS, et. al (2011), mas exporia ainda mais pessoas a exames invasivos sem necessidade.

Uma alternativa seria utilizar o PSA total como uma ponte para procedimentos mais sofisticados e com sensibilidade e especificidade maiores, principalmente por seu baixo custo. Para aqueles que apresentarem níveis de PSA inferiores a 4,0ng/mL, o exame de toque retal é imprescindível para indicar a necessidade de novos exames, como os de imagem, o que pode diminuir o número de subdiagnósticos, além da dosagem seriada de acordo com a indicação do profissional (JEMAL A, et al., 2015).

Nosso estudo não considerou a variável do exame de toque retal em conjunto com a elevação dos níveis de PSA acima de $4,0 \mathrm{ng} / \mathrm{mL}$, o que segundo a literatura, pode aumentar a sensibilidade do rastreio clínico. Além do mais, variações no refinamento do PSA são utilizados na clínica para melhorar as taxas de sensibilidade e especificidade do método sérico, como o PSA livre, relação PSA livre e total e a densidade do PSA (que depende do valor do volume da próstata) como disponível em diretriz online da Sociedade Brasileira 
de Urologia, o que não foi abordado em nosso trabalho. Além do mais, não foi avaliado o método de análise laboratorial do PSA, que pode variar de quimioluminescência a enzimaimunoensaio, que possuem taxas de sensibilidade diferentes, mas ambas acima de 95\% (GRAZIOTTIN T, et al., 2009).

A distribuição dos casos prostáticos também pode ser analisada a partir a classificação radiológica PIRADS de maneira isolada, em que estariam indicadas formalmente as biopsias para as categorias 4 e 5 , e ainda estaria sujeita a análise a categoria 3, inconclusiva, segundo Mussi TC (2018). No presente estudo, foi revelado que $71,4 \%$ dos casos com classificação 3 tiveram a benignidade, como diagnóstico histopatológico. Isso mostra que a intervenção mais invasiva de uma biópsia se mostrou necessária para apenas $28,6 \%$ desse público, o que leva a questionamentos sobre a indicação tendo apenas como base o resultado radiológico, corroborando os estudos de Mussi (Tabela 3).

Já nos pacientes com classificação 4 , que eram $69,5 \%$ dos pacientes pesquisados, o número de pessoas com diagnóstico maligno sobe para $41,7 \%$, ou seja, quase metade dos casos necessitariam de intervenção médica. Com isso entende-se que nesse patamar da classificação, haveria sim a necessidade de uma confirmação histopatológica. Isso é fortalecido quando avaliamos a alta taxa de sensibilidade $(91,58 \%)$, porém baixa taxa de especificidade (19,05\%) da RM-rp para PI-RADS iguais ou superiores a 4 no nosso estudo, o que vai de contra à própria diretriz da Sociedade Brasileira de Urologia (2011) que apresenta valores de sensibilidade e especificidade de $62 \%$ e $77 \%$, respectivamente, para a RM de forma isolada utilizando os mesmos critérios, e também contraria os achados de Kasivisvanathan V, et al. (2018), de $74 \%$ e $88 \%$, respectivamente (Tabela 4).

Tabela 3 - Distribuição dos casos prostáticos, consoante a classificação radiológica PI-RADS e o diagnóstico histopatológico de benignidade ou malignidade.

\begin{tabular}{cccc}
\hline & \multicolumn{3}{c}{ Diagnóstico Histopatológico } \\
\hline \multirow{2}{*}{ PI-RADS } & Benigno & Maligno & Total \\
\cline { 2 - 4 } & $\mathbf{N}(\%)$ & $\mathbf{N}(\%)$ & $\mathbf{N}(\%)$ \\
\hline 3 & $20(71,4)$ & $8(28,6)$ & $28(14,0)$ \\
4 & $81(58,3)$ & $58(41,7)$ & $139(69,5)$ \\
5 & $4(12,1)$ & $29(87,9)$ & $33(16,5)$ \\
\hline Total & $105(52,5)$ & $95(47,5)$ & $200(100)$ \\
\hline
\end{tabular}

Fonte: Malveira HMH, et al., 2020.

Tabela 4 - Avaliação das ressonâncias multiparamétricas de próstata comparadas ao resultado das biópsias considerando PI-RADS 4 e PI-RADS 5 como variável positiva.

\begin{tabular}{c|c|c|c|c}
\hline VP & VN & FP & FN & Total \\
\hline 87 & 20 & 85 & 8 & 200 \\
\hline
\end{tabular}

Legenda: Sensibilidade: 91,58\%; Especificidade: 19,05\%; Acurácia 53,50\%; VPP 50,58\%; VPN

71,43\%. Fonte: Malveira HMH, et al., 2020.

Quando avaliamos a população com PI-RADS 5, no nosso estudo, observamos que houve confirmação de malignidade em $87,9 \%$ dos casos, levando a entender que a acurácia dessa classificação aumenta conforme o número dado radiologicamente (Tabela 5). Ao analisar as taxas de sensibilidade e especificidade utilizando o PI-RADS 5 como corte para indicar biópsia, confirmamos essa tendência de confirmação, porém identificamos a baixa sensibilidade que esse corte determina, o que pode ser confirmado em nosso estudo tendo em vista que mais da metade das biópsias positivadas ocorreram quando a RM-rp acusava um PI-RADS inferior a 5 (Tabela 2).

Tabela 5 - Avaliação estatística das ressonâncias multiparamétricas de próstata comparadas ao resultado das biópsias considerando isoladamente PI-RADS 5 como variável positiva.

\begin{tabular}{c|c|c|c|c}
\hline VP & VN & FP & FN & Total \\
\hline 29 & 101 & 4 & 66 & 200 \\
\hline
\end{tabular}

Legenda: Sensibilidade: 30,53\%; Especificidade: 96,19\%; Acurácia 65,00\%; VPP 87,88\%; VPN 60,48\%. Fonte: Malveira HMH, et al., 2020. 
Por fim, associamos os critérios de PSA e de RM-rp, a fim de avaliar se há uma melhora dos níveis de sensibilidade e especificidade, e, se for presente, sua extensão. Considerando como resultado positivo as ressonâncias que apresentaram o PI-RADS 4 ou 5 associadas a valores de PSA $\geq 4 \mathrm{ng} / \mathrm{ml}$, foram obtidos 158 resultados, apresentando sensibilidade igual a $93,90 \%$, especificidade de $21,05 \%$, acurácia de $58,86 \%$, valor preditivo positivo de $56,20 \%$ e valor preditivo negativo de $76,19 \%$ (Tabela 6). Ao se analisar os dados obtidos nota-se uma alta taxa de sensibilidade e por isso melhor valor preditivo negativo, o que torna menor a ocorrência de falsos negativos.

Em contrapartida, pode-se observar que tais resultados demonstraram uma baixa especificidade aumentando a ocorrência de falsos positivos. Quando se elevou o corte do PI-RADS para 5, ainda associado com um PSA superior ou igual a $4 \mathrm{ng} / \mathrm{ml}$, verificou-se que a associação dessas variáveis apresentou uma elevada especificidade, cerca de $94,75 \%$, mas baixa sensibilidade, $34,15 \%$.

Tabela 6 - Avaliação estatística das ressonâncias multiparamétricas de próstata comparadas ao resultado das biópsias considerando PI-RADS 4 ou 5 e PSA $\geq 4 \mathrm{ng} / \mathrm{ml}$.

\begin{tabular}{c|c|c|c|c}
\hline VP & VN & FP & FN & Total \\
\hline 77 & 16 & 60 & 5 & 158 \\
\hline
\end{tabular}

Legenda: Sensibilidade: 93,90\%; Especificidade: 21,05\%; Acurácia 58,86\%; VPP 56,2\%; VPN 76,19\%. Fonte: Malveira HMH, et al., 2020.

Comparando-se esses resultados com um estudo realizado em São Paulo por Furini AAC, et al. (2016) que mostra valores preditivos positivos de 30 a $42 \%$ e sensibilidade de 70 a $80 \%$ ao se levar em consideração apenas os valores de PSA e os próprios resultados encontrados neste presente estudo, pode-se inferir que 0 uso da RM associada ao PSA $\geq 4 \mathrm{ng} / \mathrm{dl}$ aumenta a fidedignidade do diagnóstico por aumentar a sensibilidade e diminuir casos de falso positivo. Por outro lado, não houve grande diferença quando comparamos a RM-rp isoladamente e sua associação com PSA. Isso poderia indicar que a RM-rp isoladamente poderia ser utilizada como rastreio.

Entretanto, dadas as diferenças financeiras entre os exames, isso não é recomendado. Portanto, para que o benefício da RM-rp seja maximizado, é interessante utilizar o PSA como exame de triagem para sua solicitação, o que ao mesmo tempo aumenta a sensibilidade do rastreio e diminui possíveis custos desnecessários.

A associação PI-RADS 5 e PSA $\geq 4 \mathrm{ng} / \mathrm{ml}$ também apresentou uma variação pequena quando comparado ao PI-RADS 5 isoladamente, destacando-se a elevação de cerca de $3 \%$ na sensibilidade. Isso corrobora 0 fato de que, quanto maior o PI-RADS, maior a possibilidade de câncer, que não é tão alterado quando o associamos ao PSA, mas que a importância de sua alta especificidade é contraposta pela baixa sensibilidade. Em casos de pacientes com PI-RADS 5, com ou sem PSA alterado, então, a indicação de biópsia passa a ser importante; mas a presença de um PI-RADS inferior a 5 não é uma contraindicação a biópsia.

Portanto, em casos de PI-RADS 4 e/ou PSA entre 4 e 10ng/dl, em que a sensibilidade é alta, porém a especificidade é baixa, a indicação formal de biópsia deve se ater a outros achados clínicos e variáveis que sugiram neoplasia, fortalecendo a importância da decisão do profissional em casos considerados duvidosos. A RM-rp, além de auxiliar no aumento da sensibilidade/especificidade dos métodos de rastreio do câncer de próstata, também é fundamental no momento do diagnóstico, o que aumenta o peso de seu pedido em casos suspeitos. A biópsia prostática por via transretal, segundo Parker C, et al. (2015), apesar de útil como método diagnóstico, possui limitações associadas a um número elevado de resultados falsos negativos e iatrogenias decorrentes da técnica invasiva do exame, como hemorragias, infecções, retenção urinária, disfunção erétil e até morte.

Estudos populacionais demonstraram que, de acordo com Nam RK, et al. (2010), 4,1\% dos pacientes que realizaram esse tipo de procedimento, em um período de 30 dias desde sua realização, tiveram que ser internados devido complicações associadas. Já a biópsia guiada ressonância magnética, como preconizado por Pokorny MR, et al. (2014), permite a melhor análise da próstata e de suas patologias, implicando na melhor delimitação da área a ser biopsiada, reduzindo consequentemente o número de áreas retiradas e a 
incidência de iatrogenias. Dessa forma, a comparação entre a incidência de complicações oriundas desses métodos de biópsia prostática e sua análise é encorajada em estudos futuros uma que vez que, como limitação na pesquisa atual, não foi possível ter acesso ao laudo das biópsias a fim de verificar a presença de eventos adversos intraoperatórios.

\section{CONCLUSÃO}

A RM-rp eleva tanto a sensibilidade quanto a especificidade do diagnóstico do câncer de próstata quando comparado ao PSA de maneira isolada, mas não deve ser o primeiro exame a ser solicitado, e sim caso haja uma alteração de PSA. Seus valores de especificidade aumentam quanto maiores os níveis de PI-RADS, entretanto às custas de uma perda de sensibilidade. Em valores de PI-RADS 3 e 4, a solicitação de biópsia deve ser ponderada pelo profissional com base também na clínica e em outros parâmetros de avaliação, mas em PI-RADS 5 seu pedido é importante. Outro benefício a ser considerado, refere-se a possível diminuição de incidência de eventos indesejados durante a biópsia com a RM quando comparado à biopsia convencional.

\section{REFERÊNCIAS}

1. ALVES LS, ASSIS BPS. Câncer de próstata com PSA normal. Rev Med Minas Gerais, 2007; 17 (3/4):169-71.

2. AMERICAN COLLEGE OF RADIOLOGY. PI-RADS. Prostate Imaging - Reporting and Data System. 2015.

3. BJURLIN MA, et al. Optimization of Prostate Biopsy: the Role of Magnetic Resonance Imaging Targeted Biopsy in Detection, Localization and Risk Assessment. The Journal of Urology, 2014; 192(3), 648-658.

4. CASTRO HAS, et al. Contribuição da densidade do PSA para predizer o câncer da próstata em pacientes com valores de PSA entre 2,6 e 10,0 ng/ml. Radiol Bras, São Paulo, 2011; 44(4): 205-209.

5. EUROPEAN ASSOCIATION OF UROLOGY. Sociedade Brasileira de Urologia. Pocket Guidelines. 2018.

6. FAY AP, et al. Câncer de próstata. Doença inicial e recorrência bioquímica. Sociedade Brasileira de Oncologia Clínica, 2017; $14-16$.

7. FURINI AAC, et al. Nível sérico de antígeno prostático específico em usuários de um laboratório clínico de Novo Horizonte, São Paulo. RBAC, 2017; 49(1): 52-6.

8. GONÇALVES TN e MELLA JUNIOR SE. Avaliação do nível sérico de antígeno prostático específico (PSA) em pacientes da cidade de Campo Mourão-PR. Revista brasileira de análises clínicas, 2007; 279-281.

9. GRAZIOTTIN T, et al. Rastreamento e diagnóstico de câncer de próstata. Rev. AMRIGS, 2009; 179-183.

10. JEMAL A, et al. Prostate Cancer Incidence and PSA Testing Patterns in Relation to USPSTF Screening Recommendations. Jama, [S. I.]. 2015.

11. KASIVISVANATHAN V, et al. MRI-Targeted or Standard Biopsy for Prostate-Cancer Diagnosis. New England Journal of Medicine, 2018; 378(19): 1767-1777.

12. MINISTÉRIO DA SAÚDE. Instituto Nacional de Câncer. Versão para Profissionais de Saúde. 2019.

13. MOORE KI, et al. Anatomia Orientada Para a Clínica. 8a Ed. Rio De Janeiro: Guanabara Koogan, 2018; 373-378.

14. MORAES MC. O estudo da acurácia da ressonância magnética multiparamétrica no diagnóstico do câncer de próstata. Dissertação (Mestrado em Medicina e Ciências da Saúde). Pontifícia Universidade Católica do Rio Grande do Sul, Porto Alegre, 2017.

15. MOTTET N, et al. Professionals S. EAU Guidelines: Prostate Cancer. European Association of urology, [S. I.], 2019.

16. MULHEM E, et al. Prostate Cancer Screening. Am Fam Physician, 2015; 92(8): 683-638. MUSSI TC. A ressonância magnética multiparamétrica de próstata nos dias atuais. Einstein (São Paulo). 2018; 16(2): eMD4408.

17. NAM RK, et al. Increasing Hospital Admission Rates for Urological Complications After Transrectal Ultrasound Guided Prostate Biopsy. The Journal of Urology, 2010; 183(3), 963-969.

18. PARKER C, et al. Cancer of the prostate: ESMO Clinical Practice Guidelines for diagnosis, treatment and follow-up. Annals of Oncology, 2015; 26(suppl 5), v69-v77.

19. POKORNY MR, et al. Prospective Study of Diagnostic Accuracy Comparing Prostate Cancer Detection by Transrectal Ultrasound-Guided Biopsy Versus Magnetic Resonance (MR) Imaging with Subsequent MR-guided Biopsy in Men Without Previous Prostate Biopsies. European Urology, 2014; 66(1), $22-29$.

20. SANTOS M. Estimativa 2018: Incidência de Câncer no Brasil. Revista Brasileira de Cancerologia. 2018; 64(1):119120.

21. SOCIEDADE BRASILEIRA DE UROLOGIA. Nota oficial 2018 - Rastreamento do Câncer de Próstata. Portal da Urologia, 2019.

22. STEFFEN RE, et al. Rastreamento populacional para o câncer de próstata: mais riscos que benefícios. Physis, Rio de Janeiro, 2018; 28(2): e280209. 